\title{
OPTIMAL TRANSPORT OF NONSTATIONARY HIGH INTENSITY BEAMS"
}

\author{
$\underline{\text { A.I.Borodich }^{+}}$, ISIR, Minsk, I.A.Volkov, BSU, Minsk
}

\begin{abstract}
The problem to determine focusing field configuration that provides the minimal transverse emittance growth of an intense continuous beam at the end of the transport channel is considered. Nonlinear terminal Mayer problem of optimal control related with nonstationary space charge dominated beam transport by fringe magnetic quadrupoles was formulated. Then it was reduced to the quadrupoles parameters optimization. Squared transverse rms emittance of a beam was casted as the quality criteria. Matrix differential equations [1] for the transfer map factors together with the integral equations [1] for space charge potentials were evaluated. The solutions of the optimization problem were received by the Nelder-Mead method combined with the penalty functions. The computer code was carried out and verified. Test results are represented.
\end{abstract}

\section{INTRODUCTION}

The design of focusing systems provide the minimal transverse emittance growth of an intense relativistic beam has been one of the central challenges in charged particle beam physics and accelerator science for some decades. From the mathematical viewpoint minimizing the emittance is a problem of the optimal control theory [2]. It is formulated for a system of differential equations that govern the dynamics of an object under a control in order to find out the minimum of some functional, for example, the transverse rms emittance.

Considerable difficulties will emerge often in the optimal control theory while the minimum of a functional is calculated. Therefore, when some physical process is under the examination, one should reduce the optimal control problem to the problem of optimization, i.e. to find out the minimum of the function of many variables. For this purpose it is recommended [3] to approximate the control function by a set of independent polynomials and to use the conjugated variables for calculating the quality criteria gradient.

\section{OPTIMIZATION SCHEME}

We consider continuous nonstationary high-current beam with the Gaussian distribution as initial. The matrix differential equations for the transfer map factors

\footnotetext{
${ }^{*}$ Work supported by the FFR of Republic of Belarus, grant M96-065

+ Email: bor@isir.minsk.by
}

together with the integral equations for space charge potentials are used in frame of the algebraic approach for transverse nonlinear focusing [1]. Calculation of beam macroscopic parameters, including the transverse rms emittance, implies the Heisenberg picture in statistical mechanics. Notations of physical values, that are involved, are the same as in [1].

Let the focusing system consists of the magnetic quadrupoles cascading axially. Also the drifts may be inserted between the lenses. Scalar potential is $\varphi^{\text {field }}(x, y ; z)=0$, and the components of vector potential $\mathbf{A}^{\text {field }}(x, y ; z)$ including the fringe fields are

$$
\begin{aligned}
& A_{x}{ }^{\text {field }}(x, y ; z)=\frac{1}{4} g^{\prime}(z)\left(x^{3}-x y^{2}\right), \\
& A_{y}{ }^{\text {field }}(x, y ; z)=\frac{1}{4} g^{\prime}(z)\left(-y^{3}+x^{2} y\right), \\
& A_{z}{ }^{\text {field }}(x, y ; z)=\frac{1}{2} g(z)\left(y^{2}-x^{2}\right)- \\
& -\frac{1}{12} g^{\prime \prime}(z)\left(y^{4}-x^{4}\right),
\end{aligned}
$$

where $g(z)$ is the gradient of a single magnetic quadrupole lens, $g^{\prime}(z), g^{\prime \prime}(z)$ are its derivatives with respect to an independent variable $z$.

The Mayer's problem of optimal control is stated as: determine physical parameters of magnetic quadrupoles (magnitudes of gradients, values of the first and the second derivatives of gradients) as functions of the longitudinal coordinate $z$, which provide the minimal transverse rms emittance of an intense continues beam at the end of the transport channel.

This is nonlinear terminal problem of optimal control as the total length of the transport channel is fixed. It is reduced in the standard manner [3] to the problem of optimization of parameters of the quadrupoles and drifts.

The quality criteria (the objective functional or merit functional) is squared transverse rms emittance of a beam

$$
\begin{aligned}
Q & =<x^{2}><p_{x}^{2}>-<x p_{x}>^{2}+ \\
& +<y^{2}><p_{y}^{2}>-<y p_{y}>^{2},
\end{aligned}
$$

where the angular brackets mean averaging the physical values over an ensemble. 
Each quadrupole possesses 4 parameters: the length of a lens, the magnitude of gradient, the value of the first derivative of gradient, the value of the second derivative of gradient. Each drift possesses 1 parameter: the length of free space.

The assumption we make is that the action of each lens is independent of the others. It means we neglect the fields of one lens tend to leak into the region of any adjacent lenses [4]. Also we model the action of each lens using the "hard-edge" approximation. But both linear and nonlinear focusing forces of a lens are taking into account.

As a result of optimization 4 parameters (3 physical and 1 geometrical) for each lens plus the lengths of all drifts should be determined. The total number of parameters of optimization is $n=4 m+m-1$, where $m$ is the number of quadrupoles. We employ the Nelder-Mead method to compute the optimal values. It is the regular search method of the zero order and uses the simplex in ndimensional space of the parameters of optimization.

The general calculated scheme to obtain the optimal focusing system parameters for a transport of an intense continuous beam with the initial Gaussian distribution on coordinates and momenta looks as it follows.

1. Define the initial configuration of focusing fields, which is determined by $\mathrm{n}$ parameters, and make the initial simplex.

2. Compute the transfer map, having the dynamic equations for its factors, from the start point $z^{i n i}$ to the end $z^{f i n}$ of the transport channel.

3. Compute the second moments as the average physical values in the Heisenberg picture, using the known transfer map factors, at the end of the transport channel $z^{\text {fin }}$.

4. Calculate the terminal meaning of the quality criteria, which corresponds to the initial configuration of focusing fields.

5. Make an advanced simplex, according to the Nelder $\&$ Mead idea to move it toward the optimum, and define the advanced configuration of focusing fields.

Then we repeat actions following steps 2-5 until obtain the minimum of the quality criteria as a function of $n$ parameters with prescribed accuracy.

Three main operations are used to transfer the simplex with $(n+1)$ vertices in $n$-dimensional space. The coefficients of reflection, stretching and compression are $\alpha=1, \beta=0.5, \gamma=2$, as it is recommended in [5].

Also the additive penalty function are implemented in the optimization. It provides the beam "effective" transverse size $\left(\sim 3 \sigma_{x}^{\prime}, \sim 3 \sigma_{y}^{\prime}\right)$ is no greater than the transport channel aperture as well as the beam "effective" transverse momentum $\left(\sim 3 \lambda_{x}^{\prime}, \sim 3 \lambda_{y}^{\prime}\right)$ never exceeds the longitudinal one.

It was the outer cycle implies the regular search for the quality criteria function minimum. There is also two inner cycles to compute the transfer map in selfconsistent manner together with the space charge calculation.

The transport channel consists of the focusing elements. And every focusing element is conditionally divided with respect to the $z$ variable onto 5 sections (6 calculated points). Data received are stored for statistics to construct the empiric formulas for unknown functions $C_{i j}(z)$ and $\mathbf{D}_{i j}(z)$ within each focusing element. We use the modified method of averages to establish the type of each empiric formula and calculate its 2 parameters. The transfer map from $z^{i n i}$ to $z^{f i n}$ is computed into 2 stages.

In the first stage we deal with the sections. Every time we calculate the transfer map from the start point $z_{i}$ to the end $z_{i+1}$ of the current section $\left(z_{0}=z^{i n i}\right.$, $\left.z_{I}=z^{f i n}\right)$. All the coefficients $C_{i j}$ and $\mathbf{D}_{i j}$ are constant, and we derive the dynamic equations for the current section transfer map factors. So, the transfer map from $z^{i n i}$ to $z^{f i n}$ is a consequence of transfer maps from $z_{i}$ to $z_{i+1}$.

In the second stage we deal with the elements. Every time we calculate the transfer map from the start point $z_{k}$ to the end $z_{k+1}$ of the current element $\left(z_{0}=z^{i i}\right.$, $\left.z_{K}=z^{f n}\right)$. All the coefficients $C_{i j}(z)$ and $\mathbf{D}_{i j}(z)$ are known functions of $z$, and we derive the dynamic equations for the current element transfer map factors. So, the transfer map from $z^{n}$ to $z^{f n}$ is a consequence of transfer maps from $z_{k}$ to $z_{k+1}$.

\section{FRINGE QUADRUPOLES OPTIMIZATION}

The optimization scheme discussed above is an algorithm for computer code LIE_OPT written in Fortan-90. Matrix differential equations are computed by the Runge-KuttaMerson method of the 4-th order. Four-dimensional integrals, used to calculate the beam macroscopic parameters, are executed by the Monte-Carlo method. Two-dimensional integrals, used to calculate space charge potentials values, we evaluate by the Gauss method for hyper-rectangles.

As an example we consider the optimization of a fringe magnetic quadrupole channel without drifts.

Let an electron Gaussian beam current is $I=100 \mathrm{~A}$, a reference particle energy is $E_{0}=1 \mathrm{MeV}$, initial centroid parameters are $\bar{x}(0)=0, \bar{y}(0)=0$, initial sizes are $\tilde{x}(0)=0.25 \times 10^{-2} \mathrm{~m}, \quad \tilde{y}(0)=0.25 \times 10^{-2} \mathrm{~m}, \quad$ initial divergences are of $1 \%$.

Let a quadrupole channel consists of 10 lenses of the same length. 
The goal is to obtain the optimal values of the gradient $g$ and its second derivative $g^{\prime \prime}$ for each lens.

To facilitate our task we require each quadrupole produces the same fringe field. It means that additional constraints should be included in the penalty function.

Figure 1 depicts the initial and optimal gradient values of each quadrupole in SI units. Solid line specifies the initial focusing field configuration as a quadrupole super triplet. Corresponding variations of the beam envelopes along the transport channel in SI units is shown on figure 2 also in solid. Dashed lines on the figures 1 and 2 concern the optimal quantities.

At the end of a transport channel the initial value of squared transverse rms emittance of a beam is $Q^{0}=2.65 \times 10^{-7} \mathrm{~m} / \mathrm{rad}^{2}$, and the optimal one is $Q^{\text {opt }}=1.87 \times 10^{-7} \mathrm{~m} / \mathrm{rad}^{2}$. The optimal meaning of fringe fields is specified by $g^{\prime \prime}=-0.2 \quad \mathrm{Tl} / \mathrm{m}^{3}$. They are responsible for the partial compensation of space charge nonlinearities. It leads to some reduction of the rms emittance growth.



Figure 1: Arrangements of qudrupoles and their gradient values. .

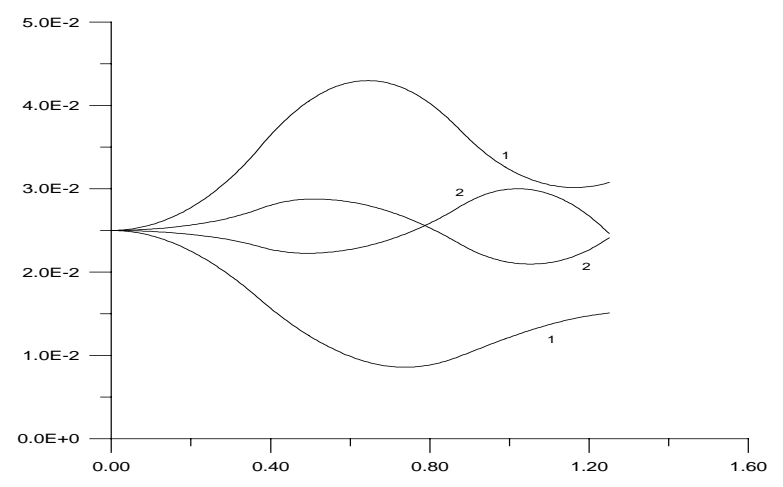

Figure 2: Beam envelopes variations.

We should also mark another nontrivial result, that follows from the solution of the optimal transport of highcurrent continuous beam. It is possible to minimize the transverse rms emittance growth without any increase of the beam sizes while using in particular the fringed magnetic quadrupoles.

\section{CONCLUSION}

The optimization technique described here may be used in computer simulations for high-current continuous beam transport to provide the minimal emittance growth.

The major advantage of this approach is that it uses the most general equations that govern the dynamics of nonstationary space charge dominated beam. Also it is important that focusing field nonlinearities may be included in the group of controls.

The major drawback is that technique searches only local minimum. Therefore in future work the procedure of picking a starting point in the space of parameters will be adjusted.

\section{REFERENCES}

[1] A.I.Borodich, "Transverse Nonlinear Focusing of Nonstationary Space Charge Dominated Beams", (this Proceedings).

[2] N.N.Moiseev, "Numerical Methods in Theory of Optimal Systems", Moscow, (1971), (in Russian).

[3] A.A.Kuraev, "Powerful Microwaves Devices: Methods of Analysis and Optimization of Parameters", Moscow, (1986), (in Russian).

[4] C.K.Allen, S.K.Guharay, M.Reiser, "Optimal Transport of Low Energy Particle Beams", Proceedings of the 1995 IEEE Particle Accelerator Conference, pp.2324-2326, (1996).

[5] B.D.Bunday, "Basic Optimization Methods", Edward Arnold, (1984) 